Ana Luisa Jiménez 1

\title{
Prevenção de doenças sexualmente transmissíveis em mulheres: associação com variáveis sócio-econômicas e demográficas
}

\author{
Prevention of sexually transmitted diseases \\ among women: association with socioeconomic \\ and demographic variables
}

Sabina Léa Davidson Gotlieb 2

Ellen Hardy 1,3

Lourens J. D. Zaneveld 4

\footnotetext{
1 Centro de Pesquisa das Doenças Materno-Infantis de Campinas.

C. P. 6181, Campinas, $S P$ 13081-970, Brasil. ajimez@mixmail.com

2 Departamento de Epidemiologia, Faculdade de Saúde Pública, Universidade de São Paulo. Av. Dr. Arnaldo 715, São Paulo, SP 01246-904, Brasil. sgotlieb@usp.br 3 Departamento de Tocoginecologia, Faculdade de Ciências Médicas, Universidade Estadual de Campinas. C. P. 6181, Campinas, $S P$ 13081-970, Brasil.

hardy@unicamp.br 4 Program for the Topical Prevention of Conception and Disease, Rush Presbyterian - St. Luke's Medical Center. 1653 West Congress Parkway, Chicago, IL 60612-3833, United States. lzaneveld@rush.edu
}

\begin{abstract}
Sexually transmitted diseases (STDs) have been a subject of discussion both among scientists and in the mass media, especially because of their association with the human immunodeficiency virus (HIV). We studied the adoption of specific protective behaviors for the prevention of STDs among women, as well as the associations between these behaviors and socioeconomic and demographic variables. This was a descriptive study based on secondary data from a previous study carried out in Campinas, São Paulo State, Brazil. A total of 635 women were selected using the social network ("snowball") technique. Subjects were classified into four groups: adolescents and adults of upper middle and lower socioeconomic status, respectively. Condoms were the STD prevention method most frequently mentioned by interviewees. A negative association was observed between having a steady partner and condom use in all the groups. The main reason mentioned for not using condoms was "having a single partner and trusting him". Among adolescents, a positive association was observed between schooling above the 8th grade and condom use, and a negative association was observed between age and condom use. Among adults, only condom use in general was also positively associated with socioeconomic status.
\end{abstract}

Key words Women; Condom; Sexually Transmitted Diseases; Socioeconomic Status

Resumo As doenças sexualmente transmissíveis (DST) têm sido debatidas no ambiente científico e nos meios de comunicação de massa, em especial, por sua associação a maior risco de infecção pelo Vírus da Imunodeficiência Humana (HIV). Estudou-se a adoção de comportamentos por mulheres para proteção das DST, tal como a associação destes a variáveis sócio-econômicas e demográficas. Trata-se de estudo descritivo, com dados secundários de pesquisa feita em Campinas, São Paulo, na qual foram entrevistadas 635 mulheres selecionadas mediante a técnica de amostragem "bola de neve". Foram classificadas em: adolescentes e adultas de status sócio-econômico médio-alto ou baixo. Grande proporção delas não se prevenia das DST, em particular, as de status baixo. Em todos os grupos, o condom masculino foi o método de prevenção mais referido. Houve associação negativa entre parceiro fixo e uso de condom, e a principal razão para não usá-lo foi "só ter um parceiro e confiar nele". Em meio às adolescentes, ocorreu associação positiva entre escolaridade acima da oitava série e uso de condom, bem como negativa entre idade e uso desse método. Entre adultas o uso exclusivo de condom esteve, em geral, positivamente associado a status sócio-econômico.

Palavras-chave Mulheres; Preservativo; Doenças Sexualmente Transmissíveis; Posição Sócioeconômica 


\section{Introdução}

Mais de vinte tipos diferentes de doenças são transmitidas através do contato sexual (O'Leary \& Cheney, 1993) e representam grave problema de saúde pública por suas repercussões médicas, sociais e econômicas. As doenças sexualmente transmissíveis (DST) ocorrem com maior freqüência nos países em desenvolvimento, onde constituem a segunda maior causa de perda de vida saudável entre mulheres de 15 a 45 anos (Dallabetta et al., 1997). Atualmente, tem sido ressaltada sua associação com maior risco de infeção pelo Vírus da Imunodeficiência Humana (HIV) (CDC, 1998).

As DST têm sido associadas à promiscuidade sexual, provocando estigma moral e social nas pessoas que as contraem, levando à deterioração de seus relacionamentos e desvalorização social. Como conseqüência, algumas mulheres optam por não procurar os devidos cuidados médicos (Van Dam, 1995; Dallabetta et al., 1997). Para as mulheres, as DST podem causar sofrimento por complicações e seqüelas decorrentes da ausência de tratamento, já que, apesar de algumas serem curáveis, a maioria dessas doenças apresenta infecções sub-clínicas ou pode ser assintomática durante muito tempo (Dallabetta et al., 1997; CDC, 1998). Nesse contexto, as DST significam sério problema para a saúde reprodutiva, pois podem causar doença inflamatória pélvica, o que possibilita a ocorrência de esterilidade, gravidez ectópica, parto prematuro, infecções puerperais, dor pélvica crônica e infecções recorrentes do trato superior (Dixon-Mueller \& Wasserheit, 1993; Dallabetta et al., 1997). Outras complicações associadas às DST são aborto, ruptura prematura de membranas, oftalmia purulenta do recém-nascido, sífilis congênita, baixo peso ao nascer e mortes perinatais (Azeze et al., 1995; Meda et al., 1997). Há ainda evidências de associação entre a infecção por Papilomavirus Humano (HPV) e maior risco de ter câncer de colo do útero (Dallabetta et al., 1997; Strickler et al., 1999).

Fatores biológicos, culturais e sócio-econômicos contribuem para a alta incidência e prevalência de DST e de infecção pelo HIV em mulheres. Na maioria das sociedades, estas têm pouco ou nenhum controle quanto às decisões relativas a quando e sob quais condições ter relação sexual, com relação ao uso do condom pelo parceiro e, menos ainda, das condutas sexuais dele (Faúndes, 1994; Van Dam, 1995; MacDonald, 1996).

Até agora, grande parte dos esforços em relação à prevenção das DST tem sido dirigida a mudar o comportamento sexual, estimulando a abstinência, a prática da monogamia e o uso do condom masculino (Badiani et al., 1997). Assim, diversas campanhas educativas têm encorajado o uso do condom pelo casal para prevenir as DST/AIDS; entretanto, tem sido observado que os casais heterossexuais com relações consideradas, por eles, fixas ou de longa duração, não adotam este método de forma regular, por não fazer parte de sua rotina de vida (Gertig, et al., 1997; Sangi-Haghpeykar et al., 1997; Agha, 1998). Uma explicação possível é que as mulheres ocupam posição secundária na relação, o que dificultaria a discussão aberta com o parceiro a respeito de sexo e modos de proteção (Rosenberg \& Gollub, 1992; Paiva, 1993; Hebling, 1997; Villela, 1997). Além disso, em um relacionamento afetivo, sexual e duradouro, que implica "conhecer" e "confiar" no marido ou companheiro, é difícil que o uso do condom seja negociado. Para algumas mulheres, a confiança no parceiro constitui o método de prevenção (Guimarães, C., 1996; Gogna, 1998).

O aumento da prevalência e da infeção pelo HIV entre as mulheres, a gravidade das conseqüências das DST para elas e sua freqüente ocorrência entre mulheres monogâmicas mostram a necessidade de uma abordagem dessas questões sob a perspectiva de gênero. O objetivo deste trabalho é verificar, entre mulheres, a adoção de comportamentos que vise à prevenção de doenças sexualmente transmissíveis e sua associação com variáveis sócio-econômicas e demográficas, detectando as mais importantes, do ponto de vista estatístico.

\section{Sujeitos e métodos}

Trata-se de um estudo descritivo, do tipo "estudo de mercado", que utiliza dados da pesquisa já realizada (Hardy et al., 1998a, 1998b, 1998c, 1998d). Foram entrevistados quatro grupos de mulheres, adolescentes e adultas, de estado sócio-econômico médio-alto e baixo. Incluíramse originalmente 155 mulheres em cada grupo, porém obteve-se número ligeiramente maior de mulheres adultas tanto de nível baixo (164) como de nível médio-alto (161), completandose um total de 635 mulheres entrevistadas. As participantes foram divididas em dois estratos etários, de acordo com a idade - adolescentes (15 a 19 anos) e adultas (20 a 45 anos) - e segundo seu status sócio-econômico - médio-alto e baixo -, isto é, segundo os critérios de classes sociais estabelecidos pela Associação Brasileira de Anunciantes (ABA)/Associação Brasileira dos Institutos de Pesquisa de Mercados 
(ABIPEME) (Almeida \& Wickerhauser, 1991). A amostra foi intencional, utilizando a técnica de "bola de neve" (Patton, 1990) pela dificuldade em conseguir acesso a mulheres de classe econômica média-alta, assim como a adolescentes sexualmente ativas mediante técnicas aleatórias por domicílio. O método consiste em identificar algumas mulheres elegíveis (“egos”) e entrevistá-las para saber se aceitam participar. A todas é solicitado que indiquem os nomes (endereços e número de telefone) de amigas ou parentes que considerem semelhantes a si mesmas. Cada mulher indicada é contatada, informada de quem indicou seu nome e convidada a participar no estudo. As mulheres entrevistadas satisfizeram os seguintes critérios de seleção: ter de 15 a 45 anos, já ter tido relações sexuais, não ser laqueada/histerectomizada e ter marido/companheiro não vasectomizado. Os dados foram coletados por meio de entrevistas individuais em que se utilizou um questionário estruturado e pré-testado. Ver maiores informações em Hardy et al. (1998a, 1998b).

As variáveis aqui consideradas foram idade, status sócio-econômico, escolaridade, cor, presença de parceiro fixo, prevenção de DST, forma de prevenção, razões para não prevenção e freqüência do uso do condom masculino na prevenção de DST. Quanto à variável cor, cabe destacar que as participantes se autoclassificaram em uma das seguintes categorias: branca, parda, negra, amarela ou indígena. As respostas foram divididas em duas categorias: brancas, que correspondeu à maioria das respostas, e não-brancas, que incluiu todas as outras categorias.

A análise estatística univariada foi feita utilizando os testes Qui-quadrado ou o Exato de Fisher (Berquó et al., 1981; Armitage \& Berry 1987), e a análise multivariada, pelo emprego de regressão logística (Hosmer \& Lemeshow, 1989). O nível de significância adotado foi de $5 \%(\alpha=0,05)$. Os programas computacionais utilizados foram: Statistical Package for the Social Sciences (SPSS Inc., 1995) e EPIINFO 6.0 (Dean, 1994).

\section{Resultados}

Tanto as adolescentes quanto as adultas de status sócio-econômico médio-alto tinham mais escolaridade do que as de status baixo (Tabela $1, \mathrm{p}<0,01$ ). Enquanto $90 \%$ das adolescentes e mais de $80 \%$ das adultas entre as mulheres de status sócio-econômico baixo tinham 8a série ou menos de escolaridade, mais de $60 \%$ das adolescentes e quase $80 \%$ das adultas de status médio-alto tinham, pelo menos, completado a primeira série do ensino médio. A alta escolaridade das adultas de status médio-alto evidencia-se pelos $43,5 \%$ delas que tinham alguma educação superior, o que foi observado em menos de $1 \%$ das adultas de status baixo.

Não houve diferenças significativas, segundo o nível sócio-econômico, quanto à percentagem de mulheres que declararam ter parceiro fixo. Um terço das adolescentes de nível baixo e um quarto das de nível médio-alto não tinham parceiro fixo, o que contrasta com os $15 \%$ das adultas nessa condição, independente do nível sócio-econômico. Uma percentagem significativa maior de mulheres se autoclassificou como brancas entre as de status médio-alto do que as de status baixo (Tabela 1).

Entre $50 \%$ e mais de $75 \%$ das mulheres nos distintos grupos declararam não fazer nada para proteger-se das DST (Tabela 2). Tanto as adolescentes quanto as adultas de status médio-alto afirmaram usar condom em percentagem maior que as de status baixo. Estas diferenças foram estatisticamente significativas. Chama a atenção o fato de que, independente do status sócio-econômico, a prevalência de uso de condom foi aproximadamente o dobro entre as adolescentes se comparadas com as adultas. Estas últimas declararam com maior freqüência usar outras formas de prevenção (Tabela 2).

\begin{tabular}{|c|c|c|c|c|}
\hline \multicolumn{5}{|c|}{$\begin{array}{l}\text { Distribuição percentual das adolescentes e adultas segundo características } \\
\text { sócio-demográficas e status sócio-econômico. Campinas, São Paulo, 1996/97. }\end{array}$} \\
\hline \multirow[t]{2}{*}{ Características } & \multicolumn{2}{|c|}{ Adolescentes } & \multicolumn{2}{|c|}{ Adultos } \\
\hline & Baixo & Médio-alto & Baixo & Médio-alto \\
\hline \multicolumn{5}{|l|}{ Escolaridade 1} \\
\hline Até a 4ạ série (fundamental) & 22,6 & 3,2 & 38,4 & 3,7 \\
\hline 5ạ a 8ạ série (fundamental) & 67,1 & 35,5 & 42,7 & 18,6 \\
\hline Médio & 10,3 & 55,5 & 18,3 & 34,2 \\
\hline Superior & - & 5,8 & 0,6 & 43,5 \\
\hline \multicolumn{5}{|l|}{ Parceiro fixo 2} \\
\hline Sim & 67,1 & 74,2 & 83,4 & 85,7 \\
\hline Não & 32,9 & 25,8 & 16,6 & 14,3 \\
\hline \multicolumn{5}{|l|}{ Cor (auto-classificação) ${ }^{1}$} \\
\hline Branca & 47,7 & 82,6 & 55,5 & 75,2 \\
\hline Não branca & 52,3 & 17,4 & 44,5 & 24,8 \\
\hline Total (n) & 155 & 155 & 164 & 161 \\
\hline
\end{tabular}

$1 \mathrm{p}<0,01$ entre as adolescentes e entre as adultas.

2 Faltou informação de 1 mulher adulta de status sócio-econômico baixo. 
O número de mulheres que comunicaram usar condom aumentou quando a pergunta foi direta, em lugar de inquirir sobre formas de prevenção quanto às DST. Ao comparar as mulheres de diferentes status sócio-econômicos com relação à freqüência de uso de condom, verificou-se novamente que a percentagem que declarou usá-lo "sempre" foi maior entre as mulheres de status médio-alto do que nas de status baixo. A diferença só foi significativa entre as adolescentes (Tabela 3). Por sua vez, a percentagem que declarou usar condom "sempre" foi cerca de duas vezes superior entre as adolescentes do que entre as adultas de seu mesmo status sócio-econômico. Não houve diferenças significativas entre diferentes grupos que declararam usar o condom "às vezes" (Tabela 3).

A Tabela 4 mostra as razões referidas pelas mulheres que declararam não se prevenir con-

Tabela 2

Porcentagem de adolescentes e adultas segundo status sócio-econômico e formas de prevenção de DST. Campinas, São Paulo, 1996/97.

\begin{tabular}{|c|c|c|c|c|}
\hline \multirow[t]{2}{*}{ Formas de prevenção } & \multicolumn{2}{|c|}{ Adolescentes } & \multicolumn{2}{|c|}{ Adultas } \\
\hline & Baixo & Médio-alto & Baixo & Médio-alto \\
\hline Condom 1 & 33,5 & 47,1 & 15,9 & 28 \\
\hline Nenhuma1 & 63,3 & 49,7 & 76,8 & 64,6 \\
\hline Outras2 & 3,2 & 3,2 & 7,3 & 7,5 \\
\hline Total (n) & 155 & 155 & 164 & 161 \\
\hline
\end{tabular}

$1 \mathrm{p}<0,05$ entre as adolescentes e entre as adultas

2 Outras: higiene/ducha, freqüenta ginecologista, procura conhecer o parceiro, não tem relação sexual estando menstruada, evita relação sexual anal.

Tabela 3

Distribuição percentual de adolescentes e adultas segundo status sócio-econômico e freqüência de uso do condom para prevenir-se de DST. Campinas, São Paulo, 1996/97.

\begin{tabular}{lcccc}
\hline \multirow{2}{*}{ Freqüência de uso } & \multicolumn{2}{c}{$\begin{array}{c}\text { Adolescentes } \\
\text { Baixo }\end{array}$} & Médio-alto & \multicolumn{2}{c}{$\begin{array}{c}\text { Adultas } \\
\text { Baixo }\end{array}$} & Médio-alto \\
\hline Nunca1 & 64,5 & 47,7 & 80,2 & 70,6 \\
Sempre1 & 23,0 & 38,4 & 12,4 & 18,8 \\
Às vezes & 12,5 & 139, & 7,4 & 10,6 \\
Total $(n)^{2}$ & 152 & 151 & 162 & 160 \\
\hline
\end{tabular}

$1 \mathrm{p}<0,01$ entre as adolescentes.

2 Faltou informação de três adolescentes de status baixo e de quatro

de médio-alto; de duas adultas de status baixo e de uma de médio-alto. tra DST para adotar essa conduta. A principal razão foi ter um só parceiro e confiar nele. Este motivo foi observado entre 70 e $95 \%$ das mulheres nos diferentes grupos, sendo a proporção maior entre as de nível médio-alto. A diferença entre as que deram esta justificativa foi significativa somente entre as adultas.

Chama a atenção o fato de que houve percentagem maior (cerca de $10 \%$ ) entre as mulheres de status baixo, que expressaram não conhecer nenhum método para se prevenir das DST ou nunca ter sido alertada sobre a necessidade de se proteger. Da mesma forma, $6 \%$ das adolescentes disseram nunca ter pensado em se prevenir. É interessante notar que a razão mencionada com menor freqüência para não se proteger foi que a mulher e/ou o parceiro não gostavam do condom (Tabela 4).

$\mathrm{Na}$ análise por regressão logística, o principal fator associado ao uso de condom em geral e "sempre" foi a presença de parceiro fixo, tanto entre adolescentes quanto entre adultas, sendo fator negativo na predição do uso deste método $(\mathrm{p}<0,001)$. Entre as adolescentes, a escolaridade maior de $8 \underline{a}$ série foi fator tão importante quanto a presença de parceiro fixo como preditor de uso de condom. Entre as adolescentes, quanto maior a idade, menor foi a prevalência de uso de condom em geral e "sempre" (Tabela 5). Entre as adultas, além da presença de parceiro fixo, apenas o status médioalto foi fator preditor de maior uso de condom em geral, mas não de uso de condom "sempre".

\section{Discussão}

Antes de proceder à discussão dos resultados, é necessário lembrar que o estudo original empregou um processo de amostragem de conveniência. Portanto, os dados referem-se a grupos específicos de mulheres e não podem ser generalizados para toda a população feminina de Campinas. Entretanto, os resultados são semelhantes aos encontrados em outras pesquisas brasileiras (Guimarães, C., 1996; Hebling, 1997; Villela, 1997).

Os resultados desta amostra confirmam o uso limitado de condom - em particular, entre as mulheres de status sócio-econômico baixo e de menor escolaridade -, apesar de serem maiores as prevalências observadas, neste estudo, na população de Campinas do que as descritas para o Brasil pela Pesquisa Nacional sobre Demografia e Saúde (PNDS) de 1996 (BEMFAM/ Macro International, 1997). Nessa pesquisa nacional, apenas $24 \%$ das mulheres de 15 a 19 anos referiram uso de condom pelo parceiro 
para prevenção das DST (Badiani et al., 1997), freqüência menor do que a aqui encontrada (47\% e $34 \%$ das adolescentes de status médioalto e baixo, respectivamente). A menor prevalência de uso entre mulheres de status baixo e com menor escolaridade mostrada neste estudo coincide com as observações do PNDS de 1996.

Riehman et al. (1998) e Piccino \& Mosher (1998) também apresentam resultados semelhantes aos aqui encontrados no tocante a mulheres de áreas economicamente menos favorecidas ou com baixos ingressos. Igualmente, Piccino \& Mosher (1998) e Toulemon \& Leridon (1998) encontraram menor proporção de uso do condom em parceiros de mulheres com menor grau de instrução.

Nossos resultados sugerem que o principal fator preditivo para não uso de condom é a presença de parceiro fixo. A importância desta variável fica reforçada pela elevada percentagem de mulheres que mencionou ter só um parceiro e confiança nele como razão para não se proteger de DST.

Não se sentir em risco, provavelmente, está associado ao pouco conhecimento que as mulheres possuem sobre o significado desse termo e acerca da possibilidade de se contaminarem, mesmo sendo monógamas. Além disso, o risco é mediado por características específicas de cada mulher e determinado por sua história de vida, subjetividade e inserção social (Barbosa \& Villela, 1996). Em última instância, é avaliado em termos da confiança e do grau de intimidade alcançado com o parceiro (Lear, 1995).

Tem sido considerado pouco provável que as mulheres pensem na possibilidade de contaminar-se com DST/HIV, pois não se consideram promíscuas, confiam e conhecem seus parceiros e têm relações sexuais envolvidas em sentimentos afetivos (Guimarães, K., 1996; Heilborn \& Gouveia, 1997). Goldstein (1996) observou que as mulheres sem vínculo marital ainda têm poder para pedir/exigir o uso do condom pelo parceiro; entretanto, após o casamento, perdem-no, mesmo sabendo da possível infidelidade do companheiro e que, em outros relacionamentos, este não se protegeria de doenças. Gogna (1998) observou que a maioria das mulheres considera desnecessário o uso de condom pelos parceiros estáveis. Associações entre tipo de relação e uso de condom (ou usá-lo "sempre") são apontadas por Agha (1998), Piccino \& Mosher (1998) e Sikkema et al. (1996).

Outro fator negativo identificado neste estudo para uso do condom foi o fato, manifestado por alta proporção de mulheres, de não saber como se proteger e nem sequer ter pensado nes-
Tabela 4

Distribuição percentual de adolescentes e adultas, segundo o status sócio-econômico e razões referidas para não se prevenir de DST. Campinas, São Paulo, 1996/97.

\begin{tabular}{lcccc}
\hline Razões & \multicolumn{2}{c}{$\begin{array}{c}\text { Adolescentes } \\
\text { Baixo1 }\end{array}$} & $\begin{array}{c}\text { Médio-alto } \\
\text { Baixo1 }\end{array}$ & $\begin{array}{c}\text { Adultas } \\
\text { Médio-alto1 }\end{array}$ \\
\hline $\begin{array}{l}\text { Só tem um parceiro/ } \\
\text { confia no parceiro } 2\end{array}$ & 71,9 & 85,7 & 80,8 & 95,0 \\
$\begin{array}{l}\text { Não conhece nenhum } \\
\text { método/nunca a alertaram }\end{array}$ & 9,4 & 5,2 & 10,4 & 4,0 \\
$\begin{array}{l}\text { Nunca pensa em se prevenir } \\
\text { Mulher/parceiro não gosta } \\
\text { de usar condom }\end{array}$ & 6,3 & 6,5 & 2,4 & 3,0 \\
$\begin{array}{l}\text { Outras } \\
\text { Total (n) }\end{array}$ & 9,4 & 2,6 & 4,0 & 2,0 \\
\hline
\end{tabular}

1 Faltou informação de duas adolescentes e de uma adulta de status baixo e de três adultas de status médio-alto.

$2 p<0,05$ para as adultas. A comparação foi feita entre as adolescentes e entre as adultas para cada uma das razões por separado.

Tabela 5

Fatores associados ao uso de condom e ao uso "sempre"1 por mulheres adolescentes e adultas. Campinas, São Paulo, 1996/97.

\begin{tabular}{|c|c|c|c|c|c|}
\hline & Coef. & $\begin{array}{l}\text { E.P. } \\
\text { Coef. }\end{array}$ & OR est. & $\begin{array}{l}\text { I.C. } 95 \% \\
\text { p/OR }\end{array}$ & $p$ \\
\hline \multicolumn{6}{|l|}{ Uso de condom } \\
\hline \multicolumn{6}{|l|}{ Adolescentes $(n=310)$} \\
\hline Presença parceiro fixo & $-1,091$ & 0,265 & 0,34 & $0,20-0,57$ & $<0,001$ \\
\hline Escolaridade (>8a série) & 0,995 & 0,271 & 2,71 & $1,59-4,60$ & $<0,001$ \\
\hline Idade da mulher (anos) & $-0,214$ & 0,098 & 0,81 & $0,67-0,98$ & 0,029 \\
\hline Constante & 3,670 & 1,655 & & & 0,027 \\
\hline \multicolumn{6}{|l|}{ Adultas $(n=324)$} \\
\hline Presença de parceiro fixo & $-2,223$ & 0,251 & 0,11 & $0,07-0,18$ & $<0,001$ \\
\hline $\begin{array}{l}\text { Status sócio-econômico } \\
\text { (médio-alto) }\end{array}$ & 0,886 & 0,282 & 2,43 & $1,40-4,21$ & $<0,002$ \\
\hline \multicolumn{6}{|l|}{ Uso "sempre" } \\
\hline \multicolumn{6}{|l|}{ Adolescentes $(n=303)^{2}$} \\
\hline Presença de parceiro fixo & $-1,056$ & 0,276 & 0,35 & $0,20-0,60$ & $<0,001$ \\
\hline Escolaridade (>8ạ série) & 1,142 & 0,294 & 3,13 & $1,76-5,57$ & $<0,001$ \\
\hline Idade da mulher (anos) & $-0,265$ & 0,109 & 0,77 & $0,62-0,95$ & 0,016 \\
\hline Constante & 3,973 & 1,829 & & & 0,030 \\
\hline \multicolumn{6}{|l|}{ Adultas $(n=321)^{2}$} \\
\hline Presença de parceiro fixo & $-2,084$ & 0,194 & 0,12 & $0,09-0,18$ & $<0,001$ \\
\hline
\end{tabular}

1 Faltou informação de 7 adolescentes e de 3 adultas.

2 sempre: $1 /$ às vezes, nunca: 0 . 
sa possibilidade. Esta informação, junto com a associação entre escolaridade e uso de condom e o menor uso entre mulheres de status baixo, evidencia que os programas educativos não têm atingido estes últimos grupos populacionais.

O conjunto das informações sugere que, em função das relações de gênero existentes, as mulheres não têm poder para negociar o uso de condom por seus parceiros e continuam confiando que estes não estão expostos à contaminação fora da união. O problema agrava-se entre as mulheres de menor nível sócio-econômico e escolaridade pela falta de informação adequada.

É, portanto, fundamental promover atividades que enfatizem a necessidade da reflexão conjugal sobre os riscos de infectar-se com essas doenças, de modo a propiciar processos verdadeiros de mudança por parte do casal. Ao serem conduzidas ações educativas que visem à prevenção das DST, o primeiro passo deve ser uma discussão a respeito da vida privada do casal sob uma perspectiva de gênero. Sem estas precauções, pensar na estratégia de propor a dis-

\section{Agradecimentos}

À Fundação Andrew W. Mellon pelo apoio financeiro para a realização da pesquisa original e à CAPES e ao Ministério das Relações Exteriores, pela concessão da Bolsa de estudos durante o mestrado. Um reconhecimento especial para Maria Helena Sousa, pelo apoio estatístico. seminação ampla do uso do condom - em especial, entre pessoas com relações estáveis ou monogâmicas - parece um tanto irreal e ineficiente.

$\mathrm{O}$ aspecto mais positivo que deriva dos resultados deste estudo é a maior prevalência de uso de condom quanto menor a idade da mulher. O uso de condom referido pelas mulheres adolescentes revela que não apenas elas, mas também seus companheiros, começaram a mudar seu comportamento. Este resultado, junto com o efeito positivo da escolaridade, indica a importância de trabalhar com as novas gerações e a potencialidade dos programas de educação sexual e gênero nas escolas. Sem dúvida que isto envolve incorporar práticas preventivas, desenvolver habilidades de negociação, mudar normas de pares e trabalhar a questão de gênero para desenvolver estratégias de empoderamento.

Como habitualmente ocorre na introdução de inovações sociais, espera-se que esta transformação comportamental venha a difundir-se entre as mulheres das esferas de menor educação e, naturalmente, na de mais idade.

\section{Referências}

AGHA, S., 1998. Sexual activity and condom use in Lusaka, Zambia. International Family Planning Perspectives, 24:32-37.

ALMEIDA, P. M. \& WICKERHAUSER, H., 1991. O Critério ABA/ABIPEME - Em Busca de uma Atualização. São Paulo: Associação Brasileira de Anunciantes/Associação Brasileira dos Institutos de Pesquisa de Mercado.

ARMITAGE, P. \& BERRY, G., 1987. Statistical Methods in Medical Research. 2nd Ed. Oxford: Blackwell Scientific Publication.

AZEZE, B.; FANTAHUN, M.; KIDAN, K. G. \& HAILE, T. 1995. Seroprevalence of syphilis amongst pregnant women attending antenatal clinics in a rural hospital in North West Ethiopia. Genitourinary Medicine, 71:347-350. 
BADIANI, R.; QUENTAL, I. \& MOREIRA-DOS-SANTOS, E., 1997. DST/AIDS e a Pesquisa Nacional sobre Demografia e Saúde: Uma Análise do Nível de Conhecimento e Comportamentos de Vulnerabilização. Rio de Janeiro: Sociedade Civil Bem-Estar Familiar no Brasil/Demographic and Health Surveys, Macro Internacional.

BARBOSA, R. \& VILLELA, W., 1996. A trajetória feminina da AIDS. In: Quebrando o Silêncio. Mulheres e AIDS no Brasil (R. Parker \& J. Galvão, org.), pp. 17-32, Rio de Janeiro: Editora Relume-Dumará.

BEMFAM/MACRO INTERNATIONAL (Sociedade Civil Bem-Estar Familiar), 1997. Pesquisa Nacional sobre Demografia e Saúde: Brasil, 1996. Rio de Janeiro: BEMFAM/Macro International.

BERQUÓ, E.; SOUZA, J. M. P. \& GOTLIEB, S. L. D., 1981. Bioestatística. 2a Ed. São Paulo: Editora Pedagógica Universitária Ltda.

CDC (Centers For Disease Control and Prevention), 1998. HIV prevention through early detection and treatment of other sexually transmitted disease United States. MMWR, 47:1-7.

DALLABETTA, G.; LYN, M.; LAGA, M. \& ISLAM, M., 1997. DST: Impacto global do problema e desafios para o controle. In: Controle da Doenças Sexualmente Transmissíveis. Manual de Planejamento e Coordenação de Programas (G. Dallabetta, M. Laga \& P. Lamptey, org.), pp. 1-22, Rio de Janeiro: Editora Te Corá.

DEAN, J., 1994. EpiInfo. Computer Program for Epidemiology. Version 6.01. Atlanta: Centers for Disease Control and Prevention.

DIXON-MUELLER, R. \& WASSERHEIT, J. N., 1993. A Cultura do Silêncio: As Infecções do Trato Reprodutivo nas Mulheres do Terceiro Mundo. São Paulo: International Women's Health Coalition.

FAÚNDES, A., 1994. Reproductive tract infections. International Journal of Gynaecology and Obstetrics, 46:181-187.

GERTIG, D.; KAPIGA, S.; SHAO, J. \& HUNTER, D., 1997. Risk factors for sexually transmitted diseases among women attending family planning clinics in Dar-Es-Salaam, Tanzania. Genitourinary Medicine, 73:39-43.

GOGNA, M., 1998. Factores psicosociales y culturales en la prevención y tratamiento de las enfermedades de transmisión sexual. Cadernos de Saúde Pública, 14(Sup. 1):81-85.

GOLDSTEIN, D., 1996. O lugar da mulher no discurso sobre AIDS no Brasil. In: Quebrando o Silêncio. Mulheres e AIDS no Brasil (R. Parker \& J. Galvão, org.), pp. 137-152, Rio de Janeiro: Editora Relume-Dumará.

GUIMARÃES, C., 1996. "Mas eu conheço ele!" In: Quebrando o Silêncio. Mulheres e AIDS no Brasil (R. Parker \& J. Galvão, org.), pp. 169-180, Rio de Janeiro: Editora Relume-Dumará.

GUIMARÃES, K., 1996. Nas raízes do silêncio: A representação cultural da sexualidade feminina e a prevenção do HIV/AIDS. In: Quebrando o Silêncio. Mulheres e AIDS no Brasil (R. Parker \& J. Galvão, org.), pp. 89-114, Rio de Janeiro: Editora Relume-Dumará.

HARDY, E.; DE PÁDUA, K. S.; JIMÉNEZ, A. L. \& ZANEVELD, L. J., 1998a. Women's preferences for vaginal antimicrobial contraceptives. I - Method- ology. Contraception, 58:233-238.

HARDY, E.; DE PÁDUA, K. S.; JIMÉNEZ, A. L. \& ZANEVELD, L. J. D., 1998b. Women's preferences for vaginal antimicrobial contraceptives. II - Preferred characteristics according to women's age and socioeconomic status. Contraception, 58: 239-244

HARDY, E.; JIMÉNEZ, A. L.; DE PÁDUA, K. S.; \& ZANEVELD, L. J., 1998c. Women's preferences for vaginal antimicrobial contraceptives. III - Choice of a formulation, applicator, and packaging. Contraception, 58:246-249.

HARDY, E.; DE PÁDUA, K. S.; JIMÉNEZ, A. L. \& ZANEVELD, L. J., 1998d. Women's preferences for vaginal antimicrobial contraceptives. IV - Attributes of a formulation that would protect from STD/AIDS. Contraception, 58:252-255.

HEBLING, E., 1997. Mulheres e AIDS: Relações de Gênero e Condutas de Risco. Dissertação de Mestrado, Campinas: Faculdade de Educação, Universidade Estadual de Campinas.

HEILBORN, M. L. \& GOUVEIA, P. F., 1997. Classes trabalhadoras, mulheres e sexualidade no contexto da AIDS. In: II Seminário Saúde Reprodutiva em Tempos de AIDS (J. Galvão, R. Barbosa \& R. Parker, org.), pp. 43-54, Rio de Janeiro: Associação Brasileira Interdisciplinar de AIDS/Instituto de Medicina Social, Universidade do Estado do Rio de Janeiro.

HOSMER, D. W. \& LEMESHOW, S., 1989. Applied Logistic Regression. New York, John Wiley \& Sons.

LEAR, D., 1995. Sexual communication in the age of AIDS: The construction of risk and trust among young adults. Social Science and Medicine, 41: 1311-1323.

MacDONALD, D. S., 1996. Notes on the socioeconomic and cultural factors influencing the transmission of HIV in Botswana. Social Science and Medicine, 42:1325-1333.

MEDA, N.; SANGARÉ, L.; LANKOANDÉ, S.; SANOU, P. T.; COMPAORÉ, P. I.; CATRAYE, J.; CARTOUX, M. \& SOUDRÉ, R. B., 1997. Pattern of sexually transmitted diseases among pregnant women in Burkina Faso, West Africa: Potential for a clinical management based on simple approaches. Genitourinary Medicine, 73:188-193.

O'LEARY, S. \& CHENEY, B., 1993. Em que medida as mulheres correm risco? In: A Tripla Ameaça: AIDS e Mulheres (S. O'Leary \& B. Cheney, org.), pp. 213234, Rio de Janeiro: Dossiê Panos.

PAIVA, V., 1993. Sexualidades adolescentes: Escolaridade, gênero e o sujeito sexual. In: Sexualidades Brasileiras (R. Parker \& R. Barbosa, org.), pp. 213234, Rio de Janeiro: Editora Relume-Dumará.

PATTON, M. Q., 1990. Qualitative Evaluation and Research Methods. 2nd Ed. Newbury Park: Sage Publications.

PICCINO, L. \& MOSHER, W., 1998. Trends in contraceptives use in the United States: 1982-1995. Family Planning Perspectives, 30:4-10.

RIEHMAN, K. S.; SLY, D. F.; SOLER, H.; EBERSTEIN, I. W.; QUADAGNO, D. \& HARRISON, D. F., 1998. Dual - Method use among an ethnically diverse group of women at risk of HIV infection. Family Planning Perspectives, 30:212-217.

ROSENBERG, M. J. \& GOLLUB, E. L., 1992. Commen- 
tary: Methods women can use that may prevent sexually transmitted disease, including HIV. American Journal of Public Health, 82:1473-1478.

SANGI-HAGHPEYKAR, H.; POINDEXTER, A. \& BATEMAN, L., 1997. Consistency of condom use among users of injectable contraceptives. Family Planning Perspectives, 29:67-69.

SIKKEMA, K. J.; HECKMAN, T. G.; KELLY, J. A.; ANDERSON, E. S.; WINETT, R. A.; SOLOMON, L. J.; WAGSTAFF, D. A.; ROFFMAN, R. A.; PERRY, M. J.; CARGILL, V.; CRUMBLE, D. A.; FUQUA, R. W.; NORMAN, A. D. \& MERCER, M. B., 1996. HIV risk behaviors among women living in low-income, inner-city housing developments. American Journal of Public Health, 86:1123-1128.

SPSS INCORPORATION, 1995. SPSS for Windows. Statistical Package for the Social Sciences. Release 7.0. Chicago: SPSS Inc.

STRICKLER, H.; KIRK, G.; FIGUEROA, P.; WARD, E.; BRAITHWAITE, A.; ESCOFFERY, C.; DRUMMOND,
J.; GOEBEL, B.; WATERS, D.; McCLIMENS, R. \& MANNS, A., 1999. HPV 16 antibody prevalence in Jamaica and the United States reflects differences in cervical cancer rates. International Journal of Cancer, 80:339-344.

TOULEMON, L. \& LERIDON, H., 1998. Contraceptive practices and trend in France. Family Planning Perspectives, 30: 114-120.

VAN DAM, C., 1995. HIV, STD and their current impact on reproductive health: The need for control of sexually transmitted diseases. International Journal of Gynaecology and Obstetrics, 50(Sup. 2):121-129.

VILLELA, W., 1997. Práticas de saúde, gênero e prevenção de HIV/AIDS. In: II Seminário Saúde Reprodutiva em Tempos de AIDS (J. Galvão, R. Barbosa \& R. Parker, org.), pp. 66-72, Rio de Janeiro: Associação Brasileira Interdisciplinar de AIDS/ Instituto de Medicina Social, Universidade Estadual do Rio de Janeiro. 